\title{
$o$-WEAKLY COMPACT MAPPINGS OF RIESZ SPACES
}

\author{
BY
}

P. G. DODDS

ABSTRACT. A characterization is given of linear mappings from a Riesz space to a Banach space which map order intervals to relatively weakly compact sets. The characterization is based on recent results of Burkinshaw and Fremlin. A number of applications are made to known results concerning weakly compact mappings and to results in the theory of Banach space-valued measures.

1. Introduction. There are many results in the literature dealing with properties of continuous linear mappings of spaces of type $C(K)$ to a Banach space ([3], [13], [22], [23]). These results are often proved via the theory of Banach space-valued measures using theorems of Riesz representation type to relate the mapping to the measure. Accordingly there is an extensive treatment of Banach space-valued measures to which the $C(K)$-operator theory is closely linked [25]. It is our present purpose to show that the theory of Riesz spaces is a natural domain for a unified approach to many of the basic results from $C(K)$-operator theory and their parallel vector measure relatives without using the bridge of representation theory and it is our intention to highlight the role played by the natural lattice structure in the hope that some fresh insight will be provided.

Our approach finds its roots in the work of Kluvanek [17] who gives a theory of a Banach space-valued Daniell integral defined on a Riesz space of functions. Of importance in his work is the isolation of a property called saturability which essentially guarantees that the dominated convergence theorem of Lebesgue holds when the elementary Daniell integral is suitably extended. In the case that the Riesz space of functions is the space of real-valued continuous functions of compact support in a locally compact Hausdorff space, the notion of saturability is equivalent to the requirement that the elementary Daniell integral should map each order interval of the Riesz space to a relatively weakly compact subset of the Banach space in which the integral takes its values. Our approach then is to consider the general setting of a linear map $A: L \rightarrow Y$ of an Archimedean-Riesz space $L$ to a Banach space $Y$ which maps order intervals of $L$ to bounded subsets of $Y$. Our basic result is Theorem 4.2 which gives a number of necessary and sufficient conditions that $A$ should map order intervals of $L$ to

Received by the editors August 20, 1972 and, in revised form, December 10, 1974. AMS (MOS) subject classifications (1970). Primary 46A40, 28A30; Secondary 28A45, 46Exx.

Key words and phrases. Riesz spaces, locally s-bounded vector measures, disjoint sequences, $C(K)$ operator theory. 
relatively weakly compact sets in $Y$. In this connection, the reader is referred to the theorem on extension given by Kluvanek in [18]. Our proof of Theorem 4.2 reflects very strongly the measure theoretic motivation and has as its essential in. gredient the recent characterization of relatively weakly compact subsets of the order dual of an Archimedean-Riesz space given by Burkinshaw [6] and Fremlin [12] which extends the work of Kaplan [16] and which gives an exact extension of the Dieudonné-Grothendieck theorem [13] characterizing weakly compact sets of Radon measures. A second ingredient is quite naturally the Orlicz-Pettis lemma, and in this regard it is appropriate to draw the reader's attention to the author's paper [10] which gives an order-theoretic derivation of Phillips' lemma of which the Orlicz-Pettis lemma is a consequence [7]. The remaining results of the paper are derived systematically from Theorem 4.2 in the manner of [25] using variants of a lemma of Rosenthal [23]. The applications include an extension of the Dieudonné convergence theorem (Theorem 5.4), Vitali-Hahn-Saks theorems of Brooks, Jewett, Faires (Theorem 6.4), results of Bartle, Dunford, Schwartz, Grothendieck, Pelczyński on weakly compact mappings of $C(K)$ spaces and of Diestel, Faires on locally s-bounded measures (Theorem 7.1).

We do not discount the possibility that many of our results could in fact be derived from the traditional $C(K)$-operator theory which motivates the work, by the standard technique of equipping each principal order ideal of the Riesz space with a suitable order unit norm, passing to the norm completion and applying the representation theorem of Kakutani to the abstract $M$-space thereby obtained. In view of the objectives stated earlier, we have deliberately chosen to avoid this route as it would perhaps distort the perspective from which we wish to view the very results which would of necessity become tools of proof.

The author would like to thank Igor Kluvanek for bringing to his attention the paper of J. J. Uhl, Jr. [25]. The author is also grateful to Owen Burkinshaw and David Fremlin for making available their results prior to publication and for a number of helpful comments. Special thanks are also due to the referee for interesting comments on an earlier version of this paper.

2. Preliminary information. Throughout the paper $L$ will always denote an Archimedean-Riesz space with separating order dual $L^{\sim}$, and $Y$ a real Banach space. We shall use the terminology of Luxemburg and Zaanen [20], [21],

Definition 2.1. A linear map $A: L \rightarrow Y$ will be called $o$-bounded iff $\sup \{\|A f\|: 0 \leqslant|f| \leqslant e\}<\infty$ holcis for every $0 \leqslant e \in L$.

A linear map $A: L \rightarrow Y$ will be called $o$-weakly compact iff $\{A f: 0 \leqslant$ $|f| \leqslant e\} \subset Y$ is $\sigma\left(Y, Y^{*}\right)$ relatively compact for every $0 \leqslant e \in L$.

It is an immediate consequence of the definition that if $y^{*} \in Y^{*}$, the linear form, $A^{\sim} y^{*}$ defined by $\left(A^{\sim} y^{*}\right)(f)=y^{*}(A f)$ for $f \in L$, is order bounded and the 
$\operatorname{map} A^{\sim}: Y^{*} \rightarrow L^{\sim}$ is linear, where $L^{\sim}$ denotes the order dual of the Riesz space $L$. If $Y_{1}^{*}$ denotes the unit ball of $Y^{*}$ and $A: L \rightarrow Y$ is $o$-bounded, for each $0 \leqslant e \in L$ define $\rho_{A}(e)=\sup \left\{\left|A^{\sim} y^{*}\right|(e): y^{*} \in Y_{1}^{*}\right\}=\sup \{\|A f\|: 0 \leqslant|f| \leqslant e\}$ and note that the map $f \mapsto \rho_{A}(|f|)$ is a Riesz seminorm on $L$. The set of natural numbers will be denoted by $\omega$.

DEFinition 2.2. The Riesz space $L$ will be called principal component $\sigma$-complete if $L$ has the principal projection property and, for every $0 \leqslant e \in L$, the Boolean algebra of principal components of $e$ is $\sigma$-complete.

Note that if $L$ has the projection property or is Dedekind $\sigma$-complete, then $L$ is principal component $\sigma$-complete. A partially ordered set $(P, \leqslant)$ is said to have property $I$ iff for any sequences $\left\{x_{n}\right\}_{n \in \omega},\left\{y_{n}\right\}_{n \in \omega}$ in $P$ with $x_{n} \leqslant y_{m}$ for all $n$ and $m$, there exists $v \in P$ such that $x_{n} \leqslant v \leqslant y_{n}$ holds for all $n \in \omega$.

Definition 2.3. The Riesz space $L$ will be said to have property $P I$ iff $L$ has the principal projection property and for every $0 \leqslant e \in L$, the Boolean algebra of principal components of $e$ has property $I$.

Denote by $I_{L}$ the order ideal generated by the Riesz space $L$ in $L^{\sim}, \tilde{n}$, the band of normal integrals on the Riesz space $L^{\sim}$. The following result is due to Burkinshaw [6] and Fremlin [12] and constitutes the foundation of the paper.

THEOREM A. For a subset $B \subset L^{\sim}$, the following are equivalent.

(i) $\sup \left\{\left|\phi\left(f_{n}-f_{m}\right)\right|: \phi \in B\right\} \rightarrow 0$ as $m, n \rightarrow \infty$ for each monotone order bounded sequence $\left\{f_{n}\right\} \subset L$.

(ii) $\sup \left\{\left|\phi\left(f_{n}-f_{m}\right)\right|: \phi \in B\right\} \rightarrow 0$ as $m, n \rightarrow \infty$ for each monotone order bounded sequence $\left\{f_{n}\right\} \subset I_{L}$.

(iii) The solid hull of $B$ is relatively $\sigma\left(L^{\sim}, I_{L}\right)$ compact.

(iv) $B$ is $\sigma\left(L^{\sim}, L\right)$ bounded and $\sup \left\{\left|\phi\left(f_{n}\right)\right|: \phi \in B\right\} \rightarrow 0$ as $n \rightarrow \infty$ for each disjoint order bounded sequence $\left\{f_{n}\right\} \subset L^{+}$.

(v) For every system $\left\{f_{\tau}\right\} \subset I_{L}, f_{\tau} \downarrow_{\tau} 0$ in $I_{L}$ implies $\inf _{\tau} \sup \left\{|\phi|\left(f_{\tau}\right)\right.$ : $\phi \in B\}=0$.

3. Some properties of $I_{L}$. In this section, we wish to gather a number of properties of $I_{L}$. These are mostly known results, reformulated to suit our present purposes. Some proofs are included for ease of reference. Denote by $|\sigma|\left(I_{L}, L^{\sim}\right)$ the locally solid topology on $I_{L}$ defined by the collection of seminorms $f \mapsto|\phi|(|f|), f \in I_{L}, \phi \in L^{\sim}$.

Proposition 3.1. The (continuous) dual of $I_{L}$ equipped with the topology $|\sigma|\left(I_{L}, L^{\sim}\right)$ is $L^{\sim}$.

Proof. Let the linear functional $\phi$ on $I_{L}$ be $|\sigma|\left(I_{L}, L^{\sim}\right)$ continuous. It is easily verified that there exists $\psi \in L^{\sim}$ such that $|\phi(f)| \leqslant|\psi|(|f|)$ holds for $f \in I_{L}$. It follows that $\phi \in I_{L}^{\sim}$ and $\left|\phi^{+}(f)\right| \leqslant|\psi|(|f|),\left|\phi^{-}(f)\right| \leqslant|\psi|(|f|)$ hold 
for all $f \in I_{L}$. By Theorem 19.2 of [20], there exist $0 \leqslant \phi_{1}, \phi_{2} \in L^{\sim}, \tilde{n}$, such that $\phi_{1}=\phi^{+}, \phi_{2}=\phi^{-}$on $I_{L}$ and $\left|\phi_{1}(f)\right| \leqslant|\psi|(|f|),\left|\phi_{2}(f)\right| \leqslant|\psi|(|f|)$ for $f \in L^{\sim}, \tilde{n}$. It follows that $\phi_{1}, \phi_{2} \in L^{\sim}, \tilde{n}, \tilde{n}$. Since $L^{\sim}$ is perfect by Corollary 28.5 of [20], it follows that $\phi_{1}, \phi_{2} \in L^{\sim}$ and so also $\phi \in L^{\sim}$.

Proposition 3.2. If $0 \leqslant e \in L$, then $E=\{f \in L: 0 \leqslant f \leqslant e\}$ is $|\sigma|\left(I_{L}, L^{\sim}\right)$ dense in $F=\left\{g \in I_{L}: 0 \leqslant g \leqslant e\right\}$.

Proof. If $g \in F$ and $g$ does not belong to the $|\sigma|\left(I_{L}, L^{\sim}\right)$ closure of $E$, then by the separation theorem and Proposition 3.1 above, there exists $\phi \in L^{\sim}$ such that $\phi(g)>\sup \{\phi(f): 0 \leqslant f \leqslant e, f \in L\}$. It follows that $\phi^{+}(g) \geqslant \phi(g)>$ $\phi^{+}(e)$ and this is clearly a contradiction.

Notation. For a subset $M \subset I_{L}$, write

$$
\begin{aligned}
& M^{\sigma}=\left\{f \in I_{L}: \exists\left\{f_{n}\right\}_{n \in \omega} \subset M \text { with } f_{n} \uparrow_{n} f \text { in } I_{L}\right\}, \\
& M^{m}=\left\{f \in I_{L}: \exists\left\{f_{\tau}\right\} \subset M \text { with } f_{\tau} \uparrow_{\tau} f \text { in } I_{L}\right\} .
\end{aligned}
$$

$M_{\sigma}, M_{m}$ are defined similarly by replacing $\uparrow$ by $\downarrow$.

Lemma 3.3. Let $0 \leqslant e \in L, g \in I_{L}$ with $0 \leqslant g \leqslant e$ and $0 \leqslant \phi \in L^{\sim}$. Setting $E=\{f \in L: 0 \leqslant f \leqslant e\}$, there exists $g_{\phi} \in E^{\sigma}{ }_{\sigma}$ such that $\phi\left(\left|g-g_{\phi}\right|\right)=0$.

Proof. By Proposition 3.2, there exists a sequence $\left\{g_{n}\right\} \subset E$ such that $\phi\left(\left|g-g_{n}\right|\right)<2^{-n}$ for $n \in \omega$. As in [14], it is easily verified that $g_{\phi}=\bigwedge_{m} \bigvee_{n>m} g_{n}$ satisfies $g_{\phi} \in E^{\sigma}{ }_{\sigma}$ and $\phi\left(\left|g-g_{\phi}\right|\right)=0$.

The next result is due to Kaplan [15] and we omit its proof.

Proposition 3.4. Let $0 \leqslant e \in L, E=\{f \in L: 0 \leqslant f \leqslant e\}$ and $F=$ $\left\{g \in I_{L}: 0 \leqslant g \leqslant e\right\}$. Then $F=E_{\sigma}^{\sigma}{ }^{m}{ }_{m}$.

A simple consequence of Proposition 3.4 is

COROLlary 3.5. $\left(I_{L}\right)_{n}^{\sim}=L^{\sim}$.

4. Characterization of $O$-weakly compact maps. In this section we give a number of necessary and sufficient conditions for an $o$-bounded map $A: L \rightarrow Y$ to be $O$-weakly compact. The first result concerns the possiblity of extending $A$ from $L$ to $I_{L}$ and is familiar from standard integration theory. The proof of the proposition is a direct consequence of Proposition 3.4 and accordingly will be omitted.

PRoposition 4.1. Let $A: L \rightarrow Y$ be o-bounded and $\rho: I_{L} \rightarrow \mathrm{R}$ a Riesz seminorm such that $\left\{f_{\tau}\right\} \subset I_{L}, f_{\tau} \downarrow_{\tau}$ o in $I_{L}$ implies $\rho\left(f_{\tau}\right) \downarrow_{\tau} o$. If $\|A f\| \leqslant \rho(f)$ holds for each $f \in L$, then $A$ has an extension $A^{\prime}: I_{L} \rightarrow Y$ with the following properties. 
(i) If $0 \leqslant e \in L,[0, e]=\{f \in L: 0 \leqslant f \leqslant e\}$, and $g \in I_{L}$ with $0 \leqslant g \leqslant e$, then $A^{\prime} g$ belongs to the closure of $A([0, e])$.

(ii) For each $y^{*} \in Y^{*}$ and $f \in I_{L}, y^{*}\left(A^{\prime} f\right)=\left(A^{\sim} y^{*}\right)(f)$.

(iii) $\left\|A^{\prime} f\right\| \leqslant \rho(f)$ for $f \in I_{L}$.

Remark. If the hypotheses of the above Proposition 3.7 are satisfied and $A^{\sim \sim}: I_{L} \rightarrow Y^{* *}$ is the natural dual mapping to $A^{\sim}$, it is clear from (ii) that $A^{\prime}=A^{\sim \sim}$.

We come now to the main result of this section.

THEOREM 4.2. Let $A: L \rightarrow Y$ be o-bounded. The following statements are equivalent.

(i) $A$ is o-weakly compact.

(ii) $A^{\sim \sim}\left(I_{L}\right) \subset Y$.

(iii) $A^{\sim \sim}\left(L^{\sigma}\right) \subset Y$.

(iv) $\rho_{A}\left(e_{n}\right) \rightarrow 0$ as $n \rightarrow \infty$ for every order-bounded disjoint sequence $\left\{e_{n}\right\} \subset L^{+}$.

(v) Each monotone order bounded sequence in $L$ is $\rho_{A}$-Cauchy.

(vi) For each system $\left\{f_{\tau}\right\} \subset I_{L}, f_{\tau} \downarrow_{\tau} 0$ implies $\rho_{A}\left(f_{\tau}\right) \downarrow 0$.

(vii) $A^{\sim} Y_{1}^{*}$ is relatively $\sigma\left(L^{\sim}, I_{L}\right)$ compact.

Proof. (i) $\Rightarrow$ (ii). Let $0 \leqslant e \in L$ and write $[0, e]=\{f \in L: 0 \leqslant f \leqslant e\}$. Suppose $\overline{A([0, e])}$ is $\sigma\left(Y, Y^{*}\right)$ compact and let $i: Y \rightarrow Y^{* *}$ be the canonical injection. Suppose there exists $g \in I_{L}$ such that $0 \leqslant g \leqslant e$ but that $A^{\sim \sim g \notin}$ $\overline{i(A([0, e]))}$. By the separation theorem, there exists $y^{*} \in Y^{*}$ such that $y^{*}\left(A^{\sim \sim g}\right)$ $>\sup \left\{y^{*}(A f): 0 \leqslant f \leqslant e, f \in L\right\}$. It follows that $\left(A^{\sim} y^{*}\right)^{+}(g)>\left(A^{\sim} y^{*}\right)^{+}(e)$ which is a contradiction.

(ii) $\Rightarrow$ (iii) is trivial.

(iii) $\Rightarrow$ (iv). Note first that (iv) is equivalent to the (apparently) weaker condition

(iv) $)^{\prime}\left\|A e_{n}\right\| \rightarrow 0$ as $n \rightarrow \infty$ for every disjoint order bounded sequence $\left\{e_{n}\right\} \subset L^{+}$.

Suppose (iii) is satisfied and let $\left\{e_{n}\right\}_{n \in \omega}$ be an order bounded disjoint sequence in $L^{+}$. For each $y^{*} \in Y^{*}$, it follows immediately from the fact that $A^{\sim} y^{*} \in\left(I_{L}\right)_{n}^{\sim}$ that $\Sigma_{n} y^{*}\left(A e_{n}\right)=y^{*}\left(A^{\sim \sim}\left(\Sigma_{n} e_{n}\right)\right)$. Since $A^{\sim \sim}\left(\Sigma_{n} e_{n}\right) \in Y$ by assumption, it follows that the series $\Sigma_{n} A e_{n}$ is weakly subseries convergent and therefore strongly subseries convergent by the Orlicz-Pettis lemma [7] and (iv) follows from (iv)'.

The equivalences (iv) $\Leftrightarrow$ (v) $\Leftrightarrow$ (vi) $\Leftrightarrow$ (vii) are contained in Theorem $A$.

(vi) $\Rightarrow$ (ii). This implication follows from Proposition 4.1, noting that $\|A f\|$ $\leqslant \rho_{A}(f)$ holds for $f \in L$. 
(ii) $\Rightarrow$ (i). Let $0 \leqslant e \in L$ and set $F=\left\{f \in I_{L}: 0 \leqslant|f| \leqslant e\right\}$. By a result of Amemiya [1] and Corollary 3.5, $F$ is $\sigma\left(I_{L}, L^{\sim}\right)$ compact. Since $A^{\sim \sim}: I_{L} \rightarrow Y$ is $\sigma\left(I_{L}, L^{\sim}\right)$ to $\sigma\left(Y, Y^{*}\right)$ continuous, $A^{\sim \sim}(F) \subset Y$ is $\sigma\left(Y, Y^{*}\right)$ compact and the result follows. By this the chain of implications is complete and the theorem is proved.

Some of the above equivalent statements were proved independently by 0. Burkinshaw.

In order to make more transparent some applications, we recall some notions from the theory of vector measures. $R$ will denote a ring of subsets of a point set $X$. A vector measure $m: R \rightarrow Y$, where $Y$ is a Banach space, is a finitely additive $Y$-valued function on $R . \quad m$ is called locally bounded iff for every $E \in R$

$$
\|m\|(E)=\{\sup \|m(F)\|: F \subset E, F \subset R\}<\infty .
$$

A locally bounded vector measure $m: R \rightarrow Y$ is called locally $s$-bounded iff for every $F \in R, \lim _{n \rightarrow \infty}\left\|m\left(E_{n}\right)\right\|=0$ holds for every disjoint sequence $\left\{E_{n}\right\} \subset R$ with $E_{n} \subset F$ for every $n \in \omega$. Notice that this latter condition is in fact equivalent to the (apparently) stronger condition that $\lim _{n \rightarrow \infty}\|m\|\left(E_{n}\right) \rightarrow 0$ for every disjoint sequence $\left\{E_{n}\right\} \subset R$ with $E_{n} \subset F$ for every $n \in \omega$. If $m: R \rightarrow Y$ is a vector measure, let $L$ be the Riesz space of R-stép functions (standard representation) and define $A: L \rightarrow Y$ by setting

$$
A\left(\sum_{i=1}^{n} \beta_{i} \chi_{E_{i}}\right)=\sum_{i=1}^{n} \beta_{i} m\left(E_{i}\right)
$$

where $\left\{E_{i}\right\} \subset R$ and $E_{i} \cap E_{j}=\varnothing$ if $i \neq j$.

Proposition 4.3. Let $m: R \rightarrow Y$ be a vector measure, $A: L \rightarrow Y$ the corresponding map from the Riesz space $L$ of R-step functions.

(i) $R$ has property $I$ iff $L$ has property $P I$.

(ii) $R$ is a $\delta$-ring iff $L$ is principal component $\sigma$-complete.

(iii) $m$ is locally bounded iff $A$ is o-bounded.

(iv) $m$ is locally s-bounded iff $A$ is o-weakly compact.

Proof. (i), (ii) are easily verified and the proofs will be omitted.

(iii) Let $0 \leqslant e \in L$ be of the form $e=\chi_{E}, E \in R$. $\|m\|(E) \leqslant \rho_{A}\left(\chi_{E}\right)=$ $\sup \left\{y^{*}\left(\sum_{i=1}^{n} \alpha_{i} m\left(E_{i}\right)\right):\left|\alpha_{i}\right| \leqslant 1, y^{*} \in Y_{1}^{*}\right.$ and $\left\{E_{i}\right\}$ is an $R$-partition of $\left.E\right\} \leqslant$ $2\|m\|(E)$. Since any order interval in $L$ is contained in an order interval of the form $\left[-\chi_{E}, \chi_{E}\right]$, (iii) is proved.

(iv) It is clear from condition (iv) of Theorem 4.2 that $m$ is locally sbounded if $A$ is $O$-weakly compact. The converse follows from Proposition 3.2 of [10] and Theorem 4.2. 
5. On a theorem of Dieudonne. The principal result of this section is an extension of a well-known theorem of Dieudonné [9] and supplements the work of Kaplan [16]. If $0 \leqslant e \in L$, a principal component $l$ of $e$ in $I_{L}$ will be called $\sigma$ l.s.c. iff $l \in L^{\sigma}$.

LEMMA 5.1. Let $0 \leqslant e \in L, \epsilon>0$ and $\phi \in L^{\sim}$ with $\phi^{+}(e)>0$. There exists a $\sigma$ l.s.c. component $l$ of e such that $\phi^{+}(l)>\phi^{+}(e)-\epsilon$ and $\phi^{-}(l)<\epsilon$.

Proof. Choose $\delta$ such that $0<\delta<\epsilon / 2 \phi^{+}(e)$ and $\eta$ such that $\eta<\epsilon \delta$. Since $\phi^{+} \wedge \phi^{-}=0$, there exists $f \in L$ with $0 \leqslant f \leqslant e$ such that $\phi^{+}(f)>\phi^{+}(e)-$ $\epsilon / 2$ and $\phi^{-}(f)<\eta$. Write $e=l+k$ where $l$ is the $\sigma$ 1.s.c. component of $e$ in the band generated in $I_{L}$ by $(f-\delta e)^{+}$. Note that $\delta l \leqslant f$ and $f \leqslant l+\delta k$. It follows that $\delta \phi^{-}(l) \leqslant \phi^{-}(f)<\epsilon$ and that

$$
\phi^{+}(l)+\delta \phi^{+}(e) \geqslant \phi^{+}(l+\delta k) \geqslant \phi^{+}(f)>\phi^{+}(e)-\epsilon / 2 .
$$

Thus $\phi^{+}(l)>(1-\delta) \phi^{+}(e)-\epsilon / 2>\phi^{+}(e)-\epsilon$.

LEMMA 5.2. Let $0 \leqslant e \in L, \phi \in L^{\sim}$ and let $l^{\prime}$ be a $\sigma$ l.s.c. component of $e$ such that $|\phi|\left(l^{\prime}\right)>\epsilon$, for some $\epsilon>0$. There exists a $\sigma$ l.s.c. component $l^{\prime \prime}$ of $e$ such that $l^{\prime \prime} \leqslant l^{\prime}$ and $\left|\phi\left(l^{\prime \prime}\right)\right|>\epsilon / 4$.

Proof. For definiteness, assume that $\phi^{+}\left(l^{\prime}\right)>\epsilon / 2$. By Lemma 5.1 above, there exists a $\sigma$ l.s.c. component $l$ of $e$ such that $\phi^{+}(l)>\phi^{+}(e)-\epsilon / 8, \phi^{-}(l)<$ $\epsilon / 8$. Set $l^{\prime \prime}=l \wedge l^{\prime}$. Observe that $\phi^{-}\left(l \wedge l^{\prime}\right) \leqslant \phi^{-}(l)<\epsilon / 8$ and that $\phi^{+}\left(l \wedge l^{\prime}\right)=$ $\phi^{+}(l)+\phi^{+}\left(l^{\prime}\right)-\phi^{+}\left(l \vee l^{\prime}\right)>\phi^{+}(e)-\epsilon / 8+\epsilon / 2-\phi^{+}(e)=3 \epsilon / 8$. It follows that $\left|\phi\left(l^{\prime}\right)\right| \geqslant \phi^{+}\left(l \wedge l^{\prime}\right)-\phi^{-}\left(l \wedge l^{\prime}\right)>\epsilon / 4$ and the lemma follows.

Proposition 5.3. Let $B \subset L^{\sim}$. The following statements are equivalent.

(i) $\sup \left\{|\phi|\left(e_{n}\right): \phi \in B\right\} \rightarrow 0$ as $n \rightarrow \infty$ for every disjoint order-bounded sequence $\left\{e_{n}\right\} \subset L^{+}$.

(ii) For each $0 \leqslant e \in L, \sup \left\{\left|\phi\left(e_{n}\right)\right|: \phi \in B\right\} \rightarrow 0$ as $n \rightarrow \infty$ for every disjoint sequence $\left\{e_{n}\right\}$ of $\sigma$ l.s.c. components of $e$.

Proof. (ii) $\Rightarrow$ (i). Suppose (i) is not true. There exists $\epsilon>0,0 \leqslant e \in L$, a disjoint sequence $\left\{e_{n}\right\} \subset[0, e]$, and a sequence $\left\{\phi_{n}\right\} \subset B$ such that $\left|\phi_{n}\left(e_{n}\right)\right|>\epsilon$. For each $n \in \omega$ denote by $e_{n}^{\prime}$ the $\sigma$ 1.s.c. component of $e$ in the principal band generated in $I_{L}$ by $e_{n}$. Note that the sequence $\left\{e_{n}^{\prime}\right\}$ is disjoint and, for each $n \in \omega,\left|\phi_{n}\right|\left(e_{n}^{\prime}\right)>\epsilon$. By Lemma 5.2, there exists a sequence $\left\{e_{n}^{\prime \prime}\right\}$ of $\sigma$ 1.s.c. components of $e$ such that $e_{n}^{\prime \prime} \leqslant e_{n}^{\prime}$ and $\left|\phi_{n}\left(e_{n}^{\prime \prime}\right)\right|>\epsilon / 4$. Since the sequence $\left\{e_{n}^{\prime \prime}\right\}$ is clearly disjoint (ii) is not true and so (ii) $\Rightarrow$ (i).

(i) $\Rightarrow$ (ii). Note that if $l$ is a $\sigma$ l.s.c. component of $0 \leqslant e \in L$, there exists by definition a sequence $\left\{l_{n}\right\} \subset L^{+}$such that $l_{n} \uparrow_{n} l$ in $I_{L}$. The implication (i) $\Rightarrow$ (ii) is now a simple consequence of the fact that $B \subset\left(I_{L}\right)_{n}^{\sim}$. 
Notation. If $A: L \rightarrow Y$ is $O$-weakly compact, by virtue of Proposition 4.1 and Theorem 4.2, we will abuse notation and denote by $A$ rather than $A^{\sim \sim}$ the extension of $A$ to $I_{L}$.

We now come to the Dieudonné theorem (see also Thomas [24]).

THEOREM 5.4. Let $0 \leqslant e \in L$ be a strong order unit for the Riesz space $L$. Let $A_{n}: L \rightarrow Y$ be a sequence of o-weakly compact mappings and suppose that $\lim _{n \rightarrow \infty} A_{n} f$ exists for each $\sigma$ l.s.c. component $f$ of $e$. Then $\left\{e_{j}\right\} \subset L^{+}$.

(a) $\sup _{n} \rho_{A_{n}}\left(e_{j}\right) \rightarrow 0$ as $j \rightarrow \infty$ for each disjoint order bounded sequence

(b) There exists an o-weakly compact mapping $A: L \rightarrow Y$ such that $\lim _{n \rightarrow \infty} A_{n} f=A f$ holds for all $f \in I_{L}$.

Proof. (a) By Proposition 5.3 above, if (a) is not true, we may assume that there exists $\epsilon>0$, a sequence $\left\{y_{n}^{*}\right\} \subset Y_{1}^{*}$, a disjoint sequence $\left\{e_{n}\right\}_{n \in \omega}$ of $\sigma$ l.s.c. components of $e$ such that $\left|\left(A_{n}^{\sim} y_{n}^{*}\right)\left(e_{n}\right)\right|>\epsilon$ for each $n \in \omega$. Set $n_{1}=1$ and find inductively a subsequence $n_{k} \uparrow_{k}$ such that $\left\|A_{n_{k}}\left(e_{n_{k+1}}\right)\right\|<\epsilon / 2$. This is possible since $\left\|A_{n}\left(e_{j}\right)\right\| \rightarrow 0$ as $j \rightarrow \infty$ for each $n \in \omega$. Now observe that $\left|\left(A_{n_{k+1}}^{\tilde{2}}-A_{n_{k}}^{\sim}\right) y_{n_{k+1}}^{*}\left(e_{n_{k+1}}\right)\right| \geqslant \epsilon-\left\|A_{n_{k}}\left(e_{n_{k+1}}\right)\right\|>\epsilon / 2$ for all $k \in \omega$. Passing to a subsequence then and suitably changing notation, we may assume in addition that the original sequence $\left\{A_{n}\right\}$ satisfies $A_{n} f \rightarrow 0$ as $n \rightarrow \infty$ for each $\sigma$ 1.s.c. component $f$ of $e$. Observe now that for any infinite subset $N \subset \omega, \eta>0$ and $0 \leqslant \phi \in L^{\sim}$, there exists an infinite subset $N_{1} \subset N$ such that $\phi\left(\Sigma_{i \in N} e_{i}\right)<\eta$. In fact, if $\left\{N_{i}\right\}_{i \in \omega}$ is a partition of $N$ into an infinite number of infinite disjoint subsets and setting, for each $k \in \omega, f_{k}=\Sigma_{i \in N_{k}} e_{i}$, the assertion follows from the fact that $\Sigma_{k=1}^{\infty} \phi\left(f_{k}\right) \leqslant \phi(e)$. Using the above remarks, it follows by an inductive argument that there exists a subsequence $\left\{n_{k}\right\} \subset \omega$ such that

$$
\sum_{1<j<k}\left\|A_{n_{k}} e_{n_{j}}\right\|<\epsilon / 3,\left|A_{n_{k}}^{\sim} y_{n_{k}}\left(e_{n_{k}}\right)\right|>\epsilon \text { and }\left|A_{n_{k}}^{\sim} y_{n_{k}}\right|\left(\sum_{j>k} e_{n_{j}}\right)<\epsilon / 3
$$

hold for each $k \in \omega$. It follows that

$$
\begin{aligned}
& \left\|A_{n_{k}}\left(\sum_{j} e_{n_{j}}\right)\right\| \geqslant\left|A_{n_{k}}^{\sim} y_{n_{k}}^{*}\left(e_{n_{k}}\right)\right|-\sum_{1<j<k}\left\|A_{n_{k}} e_{n_{j}}\right\| \\
& -\left|A_{n_{k}}^{\sim} y_{n_{k}}\right|\left(\sum_{j>k} e_{n_{j}}\right)>\epsilon / 3 .
\end{aligned}
$$

Since $\Sigma_{j} e_{n_{j}}$ is a $\sigma$ l.s.c. component of $e$, this is a contradiction and (a) is proved.

(b) It now follows from (a) and Theorem 12.4 of Kaplan [16] that $\sup _{n} \rho_{A_{n}}(e)<\infty$. For $f \in I_{L}$, set $\rho(f)=\sup _{n} \rho_{A_{n}}(f)$. Note that $\rho$ is a Riesz seminorm on $I_{L}$ and that from (a) and Theorem $\mathrm{A}$, it follows that $f_{\tau} \downarrow_{\tau} 0$ in $I_{L}$ implies 
$\rho\left(f_{\tau}\right) \downarrow_{\tau} 0$. Let now $f \in L^{+}$. By the Freudenthal spectral theorem [21], there exists a sequence $s_{m} \uparrow_{m}$ of linear combinations of $\sigma$ 1.s.c. components of $e$ and a sequence of positive numbers $\epsilon_{m} \downarrow_{m} 0$ for which $\left|f-s_{m}\right|<\epsilon_{m} e$ for each $m \in \omega$. For $p \geqslant q$ and each $m \in \omega$

$\left\|\left(A_{p}-A_{q}\right) f\right\| \leqslant 2 \rho\left(\left|f-S_{m}\right|\right)+\left\|\left(A_{p}-A_{q}\right)\left(s_{m}\right)\right\| \leqslant 2 \rho(e) \epsilon_{m}+\left\|\left(A_{p}-A_{q}\right)\left(s_{m}\right)\right\|$.

It follows that $\lim _{n \rightarrow \infty} A_{n} f$ exists for each $f \in L$. Set $A f=\lim _{n \rightarrow \infty} A_{n} f$. It is clear that $\|A f\| \leqslant \rho(f)$ holds for $f \in I_{L}$ and from (a) it is immediate that $A: L \rightarrow Y$ is $O$-weakly compact and that $\rho_{A}(f) \leqslant \rho(f)$ holds for $f \in I_{L}$. Suppose that $f_{\tau} \uparrow_{\tau} f$ holds in $I_{L}$ and that $\lim _{n \rightarrow \infty} A_{n} f_{\tau}=A f_{\tau}$ holds for each $\tau$. From $\left\|A_{n} f-A f\right\| \leqslant\left\|A_{n} f_{\tau}-A f_{\tau}\right\|+2 \rho\left(f_{\tau}-f\right)$ for each $n \in \omega$ and each $\tau$, it follows that $\lim _{n \rightarrow \infty} A_{n} f=A f$ holds for each $f \in I_{L}$ and the proof of the theorem is complete.

6. Vitali-Hahn-Saks theorems. In order to smoothen the presentation of the results of the remaining sections we give first some lemmas, which are essentially variants of a lemma due to Rosenthal [23].

Lemma 6.1. Suppose $B \subset L^{\sim}$ is $|\sigma|\left(L^{\sim}, L\right)$-bounded but not relatively $\sigma\left(L^{\sim}, I_{L}\right)$ compact. There exists $\epsilon>0,0 \leqslant e \in L$, a disjoint sequence $\left\{e_{k}\right\} \subset$ $[0, e]$ and a sequence $\left\{\phi_{k}\right\} \subset B$ such that, for each $k \in \omega,\left|\phi_{k}\right|\left(e_{j}\right)<\epsilon / 2^{j}$ for $1 \leqslant j<k,\left|\phi_{k}\left(e_{k}\right)\right|>\epsilon$ and $\Sigma_{j>k}\left|\phi_{k}\right|\left(e_{j}\right)<\epsilon / 2^{k}$.

LEMMA 6.2. Let the Riesz space $L$ be principal component o-complete, and $B \subset L^{\sim}$ be $|\sigma|\left(L^{\sim}, L\right)$ bounded but not relatively $\sigma\left(L^{\sim}, I_{L}\right)$ compact. There exist $\epsilon>0,0 \leqslant e \in L, a$ disjoint sequence $\left\{e_{k}\right\}$ of principal components of $e$ and $a$ sequence $\left\{\phi_{k}\right\} \subset B$ such that for each $k \in \omega,\left|\phi_{k}\right|\left(e_{j}\right)<\epsilon / 2^{j}$ for $1 \leqslant j<k$, $\left|\phi_{k}\left(e_{k}\right)\right|>\epsilon$ and $\left|\phi_{k}\right|\left(\Sigma_{j>k} e_{j}\right) \leqslant \epsilon / 2^{k}$ where $\Sigma_{j>k} e_{j}$ is taken in the Boolean algebra of principal components of $e$.

LEMmA 6.3. (a) Let the Riesz space $L$ have property I and let $B \subset L^{\sim}$ be $|\sigma|\left(L^{\sim}, L\right)$ bounded but not relatively $\sigma\left(L^{\sim}, I_{L}\right)$ compact. There exists $\epsilon>0$, $0 \leqslant e \in L$, a disjoint sequence $\left\{e_{k}\right\} \subset[0, e]$, a sequence $\left\{f_{k}\right\} \subset[0, e]$ with $f_{k} \downarrow_{k}$ and $a$ sequence $\left\{\phi_{k}\right\} \subset B$ such that, for each $k \in \omega$,

(i) $f_{k} \wedge e_{j}=0$ for $1 \leqslant j \leqslant k$, and $\left\{e_{j}: j>k\right\} \subset\left[0, f_{k}\right]$.

(ii) $\left|\phi_{k}\right|\left(e_{j}\right)<\epsilon / 2^{j}$ for $1 \leqslant j<k,\left|\phi_{k}\left(e_{k}\right)\right|>\epsilon$, and $\left|\phi_{k}\right|\left(f_{k}\right)<\epsilon / 2^{k}$.

(b) Let the Riesz space $L$ have property PI and let $B \subset L^{\sim}$ be $|\sigma|\left(L^{\sim}, L\right)$ bounded but not $\sigma\left(L^{\sim}, I_{L}\right)$ compact. The same conclusion as in (a) above holds, provided the sequences $\left\{e_{k}\right\},\left\{f_{k}\right\}$ are replaced by sequences of principal components of $e$.

We will give only the proof of Lemma 6.3 (a). The details of the other cases are essentially similar and perhaps slightly more straightforward. 
Proof of Lemma 6.3 (a). Assume that $L$ has property $I$ and that $B \subset L^{\sim}$ is $|\sigma|\left(L^{\sim}, L\right)$ bounded but not relatively $\sigma\left(L^{\sim}, I_{L}\right)$ compact. By Theorem A, there exists $\epsilon>0,0 \leqslant e \in L$, a disjoint sequence $\left\{e_{n}\right\} \subset[0, e]$ and a sequence $\left\{\phi_{n}\right\} \subset B$ with $\left|\phi_{n}\left(e_{n}\right)\right|>\epsilon$ for each $n \in \omega$. Suppose for $1 \leqslant j \leqslant k$ there have been defined integers $n_{j} \uparrow_{j}$, infinite subsets $N_{j} \subset \omega$ with $N_{j} \downarrow_{j}$ and elements $f_{j} \in L$ with $f_{j} \downarrow_{j}$ such that $n_{k} \in N_{k-1}, f_{k} \wedge e_{n_{j}}=0$ for $1 \leqslant j \leqslant k,\left\{e_{j}: j \in N_{k}\right\} \subset$ $\left[0, f_{k}\right],\left|\phi_{n_{k}}\right|\left(e_{n_{j}}\right)<\epsilon / 2^{j}$ for $1 \leqslant j<k$ and $\left|\phi_{n_{k}}\right|\left(f_{k}\right)<\epsilon / 2^{k}$. Let $N_{k}=$ $N_{k}^{\prime} \cup N_{k}^{\prime \prime}$ be a partition of $N_{k}$ into disjoint infinite subsets $N_{k}^{\prime}, N_{k}^{\prime \prime}$. There exists $n_{k+1} \in N_{k}^{\prime}$ such that $\left|\phi_{j}\right|\left(e_{n_{k+1}}\right)<\epsilon / 2^{k}$ for infinitely many $j \in N_{k}^{\prime \prime}$. In fact, if this is not true, the $|\sigma|\left(L^{\sim}, L\right)$ boundedness of $B$ is easily contradicted. By a change of notation then, we may assume that there exists $n_{k+1} \in N_{k}^{\prime}$ such that $\left|\phi_{j}\right|\left(e_{n_{k+1}}\right)<\epsilon / 2^{k}$ for all $j \in N_{k}^{\prime \prime}$. By Lemmas 4.3 and 4.4 of [10], there exists an infinite subset $N_{k+1} \subset N_{k}^{\prime \prime}, f_{k+1} \in L^{+}$with $f_{k+1} \leqslant f_{k}, f_{k+1} \wedge e_{n_{k+1}}=0$, $\left\{e_{j}: j \in N_{k+1}\right\} \subset\left[0, f_{k+1}\right]$ and $\left|\phi_{n_{k+1}}\right|\left(f_{k+1}\right)<\epsilon / 2^{k+1}$. This completes the induction step and the lemma follows by an obvious change of notation.

We now state the main result of this section. It extends the classical HahnVitali-Saks theorem, and results of [2], [5], [10]. A similar result for vector measures has been announced by Faires [11]

THEOREM 6.4. Let the Riesz space $L$ have property I or property PI. Let $A_{n}: L \rightarrow Y$ be a sequence of o-weakly compact maps and suppose $A f=$ $\lim _{n \rightarrow \infty} A_{n} f$ exists for each $f \in L$. Then $\left\{e_{j}\right\} \subset L^{+}$.

(a) $\sup _{n} \rho_{A_{n}}\left(e_{j}\right) \rightarrow 0$ as $j \rightarrow \infty$ for every order bounded disjoint sequence

(b) $A: L \rightarrow Y$ is o-weakly compact.

(c) $A_{n} f \rightarrow A f$ for each $f \in I_{L}$.

Proof. Let $L$ have property $I$ and note first that $B=\left\{A_{n}^{\sim} y^{*}: n \in \omega\right.$, $\left.y^{*} \in Y_{1}^{*}\right\}$ is $\sigma\left(L^{\sim}, L\right)$ bounded and hence $|\sigma|\left(L^{\sim}, L\right)$ bounded by Theorem 4.6 of [10]. It follows immediately that $A: L \rightarrow Y$ is o-bounded. Suppose that (a) is not true. As in the proof of Theorem 5.4 above, we may assume in addition that $A_{n} f \rightarrow 0$ as $n \rightarrow \infty$ for each $f \in L$ but that (a) is still not satisfied. By Lemma 6.3 (a) and again passing to a subsequence if necessary we may assume that there exists $\epsilon>0,0 \leqslant e \in L$, a disjoint sequence $\left\{e_{k}\right\} \subset[0, e]$, a sequence $\left\{f_{k}\right\} \subset$ $[0, e]$ with $f_{k} \downarrow_{k}$ and a sequence $\left\{y_{k}^{*}\right\} \subset Y_{1}^{*}$ such that (i) $f_{k} \wedge e_{j}=0$ for $1 \leqslant$ $j \leqslant k,\left\{e_{j}: j>k\right\} \subset\left[0, f_{k}\right]$ for each $k \in \omega$ and (ii) $\left|A_{k}^{\sim} y_{k}^{*}\right|\left(e_{j}\right)<\epsilon / 2^{j}$ for $1 \leqslant$ $j \leqslant k,\left|\left(A_{k}^{\sim} y_{k}^{*}\right)\left(e_{k}\right)\right|>2 \epsilon$ and $\left|A_{k}^{\sim} y_{k}^{*}\right|\left(f_{k}\right)<\epsilon / 2^{k}$ for each $k \in \omega$. Note that $e \geqslant e_{1}$ $\vee \cdots \vee e_{m} \vee f_{m} \geqslant e_{1} \vee \cdots \vee e_{n}$ holds for every $m, n$ and so, by property $I$, there exists $f \in[0, e]$ with $e_{1} \vee \cdots \vee e_{n} \vee f_{n}=e_{1}+\cdots+e_{n}+f_{n} \geqslant f \geqslant$ $e_{1}+\cdots+e_{n}$ for every $n \in \omega$. Note that, for each $k,\left|f-e_{1} \vee \cdots \vee e_{k}\right|$ $\leqslant f_{k}$ and so 


$$
\begin{aligned}
\left\|A_{k} f\right\| & \geqslant\left|\left(A_{k}^{\tilde{y}} y_{k}^{*}\right)(f)\right| \\
& \geqslant\left|\left(A_{k}^{\tilde{k}} y_{k}^{*}\right)\left(e_{k}\right)\right|-\left|A_{k}^{\tilde{k}} y_{k}^{*}\right|\left(f_{k}\right)-\sum_{1 \leqslant j<k}\left|A_{k}^{\tilde{y}} y_{k}^{*}\right|\left(e_{j}\right)>\epsilon .
\end{aligned}
$$

This is clearly a contradiction and (a) is proved. (b) follows immediately from (a) and (c) follows exactly as in part (b) of Theorem 5.4.

If $L$ has property $P I$ rather than property $I$, the proof remains essentially identical upon appeal to Lemma 6.3 (b) in place of Lemma 6.3 (a).

It should be noted that (b) of the above theorem is not true if $L$ is merely assumed to have the principal projection property. In fact, let $L$ be the Riesz space of sequences which are eventually constant and let $\Sigma a_{n}$ be a conditionally convergent but not absolutely convergent series of real numbers. For $n \in \omega, f=$ $\left(f_{k}\right) \in L$, define $\phi_{n}(f)=\Sigma_{k=1}^{n} a_{k} f_{k}, \phi(f)=\Sigma_{k=1}^{\infty} a_{k} f_{k}$. Observe that $\phi_{n}(f)$ $\rightarrow \phi(f)$ for every $f \in L$, that $\phi_{n} \in L^{\sim}$ but that $\phi \notin L^{\sim}$.

\section{Sufficient conditions for $O$-weak compactness.}

TheOREM 7.1. Let $A: L \rightarrow Y$ be o-bounded. Each of the following statements is sufficient for $A$ to be o-weakly compact.

(i) No subspace of $Y$ is isomorphic to $c_{0}$.

(ii) $L$ is principal component $\sigma$-complete and no subspace of $Y$ is isomorphic to $l^{\infty}$.

Proof. We will prove only (ii) as the proof of (i) is similar. Suppose then that $L$ is principal component $\sigma$-complete and that $A: L \rightarrow Y$ is $o$-bounded but not $O$-weakly compact. By Lemma 6.2 and Theorem 4.2, there exists $\delta>0,0 \leqslant$ $e \in L$, a disjoint sequence $\left\{e_{n}\right\}$ of principal components of $e$ and a sequence $\left\{y_{n}^{*}\right\}$ $\subset Y_{1}^{*}$ such that, for each $n \in \omega,\left|\left(A^{\sim} y_{n}^{*}\right)\left(e_{n}\right)\right|>\delta$ and $\left|\left(A^{\sim} y_{n}^{*}\right)\right|\left(\Sigma_{j \neq n} e_{j}\right)<\delta / 2$, where the sum is taken in the Boolean algebra of principal components of $e$ rather than in $I_{L}$. If $\alpha=\left(\alpha_{k}\right) \in l^{\infty}$ is finitely valued write $\alpha=\sum_{i=1}^{k} \beta_{i} \chi_{S_{i}}$ where $S_{i} \in 2^{\omega}$ satisfy $S_{i} \cap S_{j}=\varnothing$ for $i \neq j$, and set $T(\alpha)=\Sigma_{i=1}^{k} \beta_{i} A\left(\Sigma_{j \in S_{i}} e_{j}\right)$. For $y^{*} \in Y_{1}^{*}$

$$
\left|y^{*}(T(\alpha))\right| \leqslant \sum_{i=1}^{k}\left|\beta_{i}\right|\left|\left(A^{\sim} y^{*}\right)\left(\sum_{j \in S_{i}} e_{j}\right)\right| \leqslant\|\alpha\|_{\infty}\left|A^{\sim} y^{*}\right|(e) \leqslant\|\alpha\|_{\infty} \rho_{A}(e) .
$$

Moreover, if $n \in \omega$, writing $\alpha_{n}=\beta_{l}$ for some $1 \leqslant l \leqslant k$,

$$
\begin{aligned}
\|T(\alpha)\| & \geqslant\left|y_{n}^{*}(T(\alpha))\right| \geqslant\left|\beta_{l}\right|\left|\left(A^{\sim} y_{n}^{*}\right)\left(e_{n}\right)\right|-\|\alpha\|_{\infty}\left|A^{\sim} y_{n}^{*}\right|\left(\sum_{j \neq n} e_{j}\right) \\
& \geqslant\left|\beta_{l}\right| \delta-\|\alpha\|_{\infty} \delta / 2 .
\end{aligned}
$$

It follows that $(\delta / 2)\|\alpha\|_{\infty} \leqslant\|T(\alpha)\| \leqslant \rho_{A}(e)\|\alpha\|_{\infty}$ so that $T$ extends to a topological isomorphism of $l^{\infty}$ into $Y$ and the proof is complete.

Part (a) of the above theorem contains results of Grothendieck [13], Bartle, 
Dunford and Schwartz [3], and Pelczyński [22]. Part (b) contains a result of Diestel and Faires [8]. Our method of proof follows Uhl [25].

The next result is related to certain results of Grothendieck [13].

THEOREM 7.2. (i) If $A: L \rightarrow Y$ is o-weakly compact whenever $A$ is o-bounded and $Y$ is a separable Banach space, then every sequence in $L^{\sim}$ which is $|\sigma|\left(L^{\sim}, L\right)$ bounded and $\sigma\left(L^{\sim}, L\right)$ convergent is also $\sigma\left(L^{\sim}, I_{L}\right)$ convergent.

(ii) If every $\sigma\left(L^{\sim}, L\right)$ convergent sequence is also $\sigma\left(L^{\sim}, I_{L}\right)$ convergent, then every o-bounded linear map $A: L \rightarrow Y$ is o-weakly compact if $Y$ is a separable Banach space.

Proof. (i) Let $\left\{\phi_{n}\right\} \subset L^{\sim}$ satisfy $\phi_{n} \rightarrow 0, \sigma\left(L^{\sim}, L\right)$ and suppose that $\left\{\phi_{n}\right\}$ is | $\sigma \mid$-bounded. Define $A: L \rightarrow c_{0}$ via $A x=\left\{\phi_{n}(x)\right\}$. That $A$ is o-bounded follows from the $|\sigma|$-boundedness of the $\left\{\phi_{n}\right\}$. Note that $A^{\sim} y_{n}^{*}=\phi_{n}$ where $y_{n} \in l^{1}$ is the sequence $\left(y_{n}\right)_{k}=\{1$ if $k=n ; 0$ if $k \neq n\}$. Since $c_{0}$ is separable, $A$ is $o$ weakly compact and consequently $B=\left\{A^{\sim} y^{*}: y^{*} \in l^{1},\left\|y^{*}\right\| \leqslant 1\right\}$ is $\sigma\left(L^{\sim}, I_{L}\right)$ relatively compact. Thus $\left\{\phi_{n}\right\}$ is $\sigma\left(L^{\sim}, I_{L}\right)$ relatively compact and so $\phi_{n} \rightarrow 0$, $\sigma\left(L^{\sim}, I_{L}\right)$.

(ii) Let $Y$ be separable and $A: L \rightarrow Y$ o-bounded but not $o$-weakly compact. By Theorem $A$ and Theorem 4.2 it follows that there exists a sequence $\left\{y_{n}^{*}\right\}$ $\subset Y_{1}^{*}$ such that no subsequence of $\left\{A^{\sim} y_{n}^{*}: n \in \omega\right\}$ is relatively $\sigma\left(L^{\sim}, I_{L}\right)$ compact. However since $Y$ is separable, the unit ball of $Y^{*}$ is a compact metric space in the $\sigma\left(Y^{*}, Y\right)$ topology. It follows that $\left\{A^{\sim} y_{n}^{*}: n \in \omega\right\}$ contains a subsequence converging pointwise on $L$ and hence by assumption on $I_{L}$ and this is a contradiction.

COROLLARY 7.3. Let the Riesz space $L$ have property I or property PI. If $Y$ is separable, then every o-bounded mapping $A: L \rightarrow Y$ is o-weakly compact.

Proof. The corollary follows immediately from Theorem 6.4.

It should perhaps be noted that the conclusion of the above corollary is not valid if $L$ has merely the principal projection property. In fact, let $L$ be the Riesz space of all sequences $f=\left\{f_{k}\right\}$ which are eventually constant, $Y$ the Banach space of all convergent sequences and let $A: L \rightarrow Y$ be the natural inclusion. Note that in this example, the Boolean algebra of principal components of the sequence $e=\{1,1, \ldots\}$ does not have property PI. Moreover the sequence $\left\{\phi_{n}\right\} \subset L^{\sim}$ defined by $\phi_{n}(f)=n\left(f_{n}-f_{n+1}\right)$ for $n=1,2, \ldots$ and $f=\left\{f_{k}\right\} \in L$ satisfies $\phi_{n} \rightarrow 0, \sigma\left(L^{\sim}, L\right)$ but if it were true that $\phi_{n} \rightarrow 0, \sigma\left(L^{\sim}, I_{L}\right)$, it would follow that $\left\{\phi_{n}\right\}$ is $|\sigma|$-bounded and this is clearly not the case.

The next result is an improvement of Corollary 7.3 for the case that $L$ has property $I$ and extends a result of Rosenthal [23] to the setting of Riesz spaces.

THEOREM 7.4. Let $L$ have property I. If $A: L \rightarrow Y$ is o-bounded that not o-weakly compact, then $l^{\infty}$ is a continuous linear image of a subspace of $Y$. 
Proof. Suppose $A: L \rightarrow Y$ is $o$-bounded but not $o$-weakly compact. By Lemma 6.3 (a), there exist $\epsilon>0,0 \leqslant e \in L$, a disjoint sequence $\left\{e_{k}\right\} \subset[0, e]$, a decreasing sequence $\left\{f_{k}\right\} \subset[0, e]$ and a sequence $\left\{y_{k}^{*}\right\} \subset Y_{1}^{*}$ such that, for every $k \in \omega, f_{k} \wedge e_{j}=0$ for $1 \leqslant j \leqslant k,\left\{e_{j}: j>k\right\} \subset\left[0, f_{k}\right],\left|A^{\sim} y_{k}^{*}\right|\left(e_{j}\right)<\epsilon / 2^{j}$ for $1 \leqslant j<k,\left|A^{\sim} y_{k}^{*}\left(e_{k}\right)\right|>2 \epsilon$ and $\left|A^{\sim} y_{k}^{*}\right|\left(f_{k}\right)<\epsilon / 2^{k}$. Without loss of generality, assume that $e$ is a strong order unit for $L$ and set $\phi=\Sigma_{k=1}^{\infty}\left|A^{\sim} y_{k}^{*}\right| / 2^{k}\left|A^{\sim} y_{k}^{*}\right|(e)$. Note that $\phi \in L^{\sim}$ and denote by $P_{\phi}$ the projection onto the carrier band of $\phi$ in $I_{L}$. By Proposition 2.1 of [10], observe that $P_{\phi} L \subset I_{L}$ is Dedekind $\sigma$-complete. If $\alpha=$ $\left(\alpha_{n}\right)_{n \in \omega} \in l^{\infty}$, define $T: l^{\infty} \rightarrow l^{\infty}$ via $\left(T_{\alpha}\right)_{n}=A^{\sim} y_{n}^{*}\left(\sum_{i=1}^{\infty} \alpha_{i} P_{\phi} e_{i}\right)$ where the sum is taken in the Dedekind $\sigma$-complete Riesz space $P_{\phi} L$. Now observe that for each $n \in \omega$

$$
\begin{aligned}
\left|\left(T_{\alpha}\right)_{n}\right| & \leqslant \sum_{i=1}^{n-1}\left|\alpha_{i}\right|\left|A^{\sim} y_{n}^{*}\right|\left(e_{i}\right)+\left|\alpha_{n}\right|\left|A^{\sim} y_{n}^{*}\left(e_{n}\right)\right|+\|\alpha\|_{\infty}\left|A^{\sim} y_{n}^{*}\right|\left(f_{n}\right) \\
& \leqslant\left(\epsilon+P_{A}(e)\right)\|\alpha\|_{\infty} .
\end{aligned}
$$

Further $\|T \alpha\|_{\infty} \geqslant\left|\left(A^{\sim} y_{n}^{*}\right)\left(\Sigma_{i=1}^{\infty} \alpha_{i} P_{\phi} e_{i}\right)\right| \geqslant 2\left|\alpha_{n}\right| \epsilon-\epsilon\|\alpha\|_{\infty}$. It follows that $\epsilon\|\alpha\|_{\infty} \leqslant\|T \alpha\|_{\infty} \leqslant\left(\epsilon+P_{A}(e)\right)\|\alpha\|_{\infty}$ and so $T$ is $1-1$ with closed range. Now denote by $M$ the linear subspace of those $f \in L$ for which there exists $\left(\alpha_{i}\right) \in l^{\infty}$ such that $P_{\phi} f=\Sigma_{i=1}^{\infty} \alpha_{i} P_{\phi} e_{i}$. Define now $S: A(M) \rightarrow T\left(l^{\infty}\right)$ via $(S(A f))_{n}=$ $y_{n}^{*}(A f)$. The proof is complete by noting that

$$
y_{n}^{*}(A f)=\left(A^{\sim} y_{n}^{*}\right)(f)=\left(A^{\sim} y_{n}^{*}\right)\left(P_{\phi} f\right)
$$

for each $n \in \omega$.

\section{A Dunford Pettis property.}

THEOREM 8.1. Let $L$ be an Archimedean-Riesz space and $A: L \rightarrow Y$ an o-weakly compact mapping. If the sequence $\left\{f_{n}\right\} \subset L^{+}$is order bounded and $f_{n} \rightarrow 0 \sigma\left(L, L^{\sim}\right)$, then $\left\|A f_{n}\right\| \rightarrow 0$.

Proof. Let $A: L \rightarrow Y$ be $o$-weakly compact. It follows from Theorem 4.2 (v) that the Riesz seminorm $\rho_{A}$ has property $A$ (iii) of [20]. If $0 \leqslant e \in L$, it follows from Theorem 3.2 of [6] and Theorem 4.2 (vi) that there exists $0 \leqslant$ $\phi \in L^{\sim}$ such that $0 \leqslant z_{\tau} \downarrow \leqslant e, \phi\left(z_{\tau}\right) \downarrow 0$ imply $\rho_{A}\left(z_{\tau}\right) \downarrow 0$. Let $0 \leqslant f_{n} \leqslant e$ and suppose that $f_{n} \rightarrow 0 \sigma\left(L, L^{\sim}\right)$. To show that $\left\|A f_{n}\right\| \rightarrow 0$ it is sufficient to show that $\rho_{A}\left(f_{n}\right) \rightarrow 0$. Let $\epsilon>0$ be given. Without loss of generality we may assume that $\Sigma_{n} \phi\left(f_{n}\right)<\infty$. By Lemma 43.2 of [19], there exist sequences $\left\{u_{n}\right\}$, $\left\{w_{n}\right\},\left\{z_{n}\right\}$ of positive elements of $L$ such that $f_{n}=u_{n}+w_{n}, 0 \leqslant u_{n} \leqslant z_{n}, \phi\left(z_{n}\right)$ $\downarrow 0$ and $\rho_{A}\left(w_{n}\right)<\epsilon$. Since $\rho_{A}\left(z_{n}\right) \downarrow 0$, it follows that $\lim _{n \rightarrow \infty} \rho_{A}\left(f_{n}\right) \leqslant \epsilon$ and the theorem follows.

It is easily seen that Theorem 8.1 above implies the well-known fact [13] that every weakly compact mapping of $C(K), K$ compact Hausdorff, to a Banach space $Y$ maps weakly convergent sequences to norm convergent sequences in $Y$.

The above Theorem 8.1 was also observed independently by Owen Burkinshaw. 


\section{REFERENCES}

1. I. Amemiya, On ordered topological linear spaces, Proc. Internat. Sympos. Linear Spaces (Jerusalem, 1960), Jerusalem Academic Press, Jerusalem; Pergamon, Oxford, 1961, pp. 14-23. MR 25 \#2411.

2. T. Andô, Convergent sequences of finitely additive measures, Pacific J. Math. 11 (1961), 395-404. MR 25 \#1255.

3. R. G. Bartle, N. Dunford and J. Schwartz, Weak compactness and vector measures, Canad. J. Math. 7 (1955), 289-305. MR 16, 1123.

4. C. Bessaga and A. Pelczyński, On bases and unconditional convergence of series in Banach spaces, Studia Math. 17 (1958), 151-164. MR 22 \#5872.

5. J. K. Brooks and R. S. Jewett, On finitely additive vector measures, Proc. Nat.

Acad. Sci. U.S.A. 67 (1970), 1294-1298. MR 42 \#4697.

6. O. Burkinshaw, Weak compactness in the order dual of a vector lattice, Trans. Amer. Math. Soc. 187 (1974), 183-201.

7. M. M. Day, Normed linear spaces, Ergebnisse der Mathematik und ihrer Grenzgebiete, N. F., Heft 21, Springer-Verlag, Berlin, 1958. MR 20 \#1187.

8. J. Diestel and B. Faires, On vector measures (preprint).

9. J. Dieudonné, Sur la convergence des suites de mésures de Radon, An. Acad.

Brasil. Ci. 23 (1951), 21-38. MR 13, 121.

10. P. G. Dodds, Sequential convergence in the order duals of certain classes of Riesz spaces, Trans. Amer. Math. Soc. 203 (1975), 391-403.

11. B. Faires, On Vitali-Hahn-Saks type theorems, Notices Amer. Math. Soc. 21 (1974), A-174. Abstract \#711-46-1.

12. D. H. Fremlin, Topological Riesz spaces and measure theory, Cambridge Univ. Press, New York, 1973.

13. A. Grothendieck, Sur les applications linéaires faiblement compactes d'espaces du type $C(K)$, Canad. J. Math. 5 (1953), 129-173. MR 15, 438.

14. S. Kaplan, On the second dual of the space of continuous functions, Trans. Amer. Math. Soc. 86 (1957), 70-90. MR 19, 868.

15. Closure properties of $C(X)$ in its second dual, Proc. Amer. Math. Soc. 17 (1966), 401-406. MR 32 \#6260.

16. - On weak compactness in the space of Radon measures, J. Functional Analysis 5 (1970), 259-298. MR 41 \#5932.

17. I. Kluvánek, Intégrale vectorielle de Daniell, Mat.-Fyz. Časopis Sloven. Akad. Vied. 15 (1965), 146-161. MR 38 \#295.

18. The extension and closure of vector measure, Vector and Operator Valued Measures and Applications, Academic Press, New York, 1973.

19. W. A. J. Luxemburg, Notes on Banach function spaces. XIVa, Nederl. Akad.

Wetensch. Proc. Ser. A 68 = Indag. Math. 27 (1965), 229-239. MR 32 \#6202a.

20. W. A. J. Luxemburg and A. C. Zaanen, Notes on Banach function spaces. VI-XIII, Nederl. Akad. Wetensch. Proc. Ser. A 66 = Indag. Math. 25 (1963), 655-681, 104-119; ibid. A 67 = Indag. Math. 26 (1964), 360-376, 493-543. MR 28 \#5324a, b; \#3381a, b.

21. - Riesz spaces. I, North-Holland, Amsterdam, 1971.

22. A. Pejlczyński, Projections in certain Banach spaces, Studia Math. 19 (1960), 209228. MR 23 \#A3441.

23. H. P. Rosenthal, On complemented and quasi-complemented subspaces of quotients of $C(S)$ for Stonian S, Proc. Nat. Acad. Sci. U.S.A. 60 (1968), 1165-1169. MR 37 \#6740.

24. Erik Thomas, L'intégration par rapport à une mésure de Radon vectorielle, Ann.

Inst. Fourier (Grenoble) 20 (1970), fasc. 2, 55-191.

25. J. J. Uhl, Jr., Applications of a lemma of Rosenthal to vector measures and series in Banach spaces (preprint).

DEPARTMENT OF MATHEMATICS, FLINDERS UNIVERSITY OF SOUTH AUSTRALIA, BEDFORD PARK, SOUTH AUSTRALIA, 5042 AUSTRALIA 